PROCEEDINGS OF THE

AMERICAN MATHEMATICAL SOCIETY

Volume 133, Number 10, Pages 3005-3012

S 0002-9939(05)07822-6

Article electronically published on March 31, 2005

\title{
REGULARIZATION FOR A CLASS OF ILL-POSED CAUCHY PROBLEMS
}

\author{
YONGZHONG HUANG AND QUAN ZHENG
}

(Communicated by Joseph A. Ball)

\begin{abstract}
This paper is concerned with the ill-posed Cauchy problem associated with a densely defined linear operator $A$ in a Banach space. Our main result is that if $-A$ is the generator of an analytic semigroup of angle $\geq \pi / 4$, then there exists a family of regularizing operators for such an ill-posed Cauchy problem by using the Gajewski and Zacharias quasi-reversibility method, and semigroups of linear operators.
\end{abstract}

\section{INTRODUCTION}

Let $A$ be an unbounded operator in a Banach space $X$ and let $-A$ generate an analytic semigroup $\{S(t)\}$ of angle $\alpha$, where $\pi / 4 \leq \alpha<\pi / 2$. In this paper, we consider the following Cauchy problem:

$$
u^{\prime}(t)=A u(t)(0<t \leq T), \quad u(0)=x .
$$

A continuous function $u(t)(0 \leq t \leq T)$ is said to be a solution of (1.1) if for $0<t \leq T, u(t)$ is continuously differentiable, its values belong to the domain of $A$, and (1.1) is satisfied.

It is not hard to show that $S(t) u(t)=x$ if $u(t)$ is a solution of (1.1). Since $S(t)$ is invertible for each $t \geq 0$ (cf. [5] p. 69]), we obtain $u(t)=S(t)^{-1} x$ for $0 \leq t \leq T$. However, $S(t)^{-1}(t \geq 0)$ is not a family of bounded linear operators. Thus (1.1) is not stable. The problem (1.1) can then lead to the general ill-posed problem, which was introduced by Tikhonov (see 10]).

If there exists a family of regularizing operators for (1.1) (cf. Definition 3.1), then the regularization of the ill-posed problem (1.1) is obtained (cf. 10]). We in this paper obtain the regularization of (1.1) by using the solution of the well-posed Cauchy problem

$$
u_{\varepsilon}^{\prime}(t)+\varepsilon A u_{\varepsilon}^{\prime}(t)=A u_{\varepsilon}(t)(0<t \leq T), \quad u_{\varepsilon}(0)=x
$$

to approximate the solution of (1.1), where $\varepsilon>0$.

This method is called the quasi-reversibility method, introduced by Gajewski and Zacharias 2] in Hilbert space at first. Showalter [9] then generalized their results to

Received by the editors December 11, 2003 and, in revised form, May 18, 2004.

2000 Mathematics Subject Classification. Primary 47A52; Secondary 47D06, 34G10.

Key words and phrases. Ill-posed Cauchy problem, quasi-reversibility, regularizing family, analytic semigroup.

This project was supported by TRAPOYT, the National Science Foundation of China (Grant No. 10371046).

(C)2005 American Mathematical Society Reverts to public domain 28 years from publication 
the case that $-A$ generates an analytic contraction semigroup of angle $\alpha$ with $\pi / 4 \leq$ $\alpha<\pi / 2$ in Hilbert space. In [9], the key point for using the approximate theorem of semigroups is to use the properties of the dissipative operator in Hilbert space. However, it is false in Banach space even if $\{S(t)\}$ is contractive. We overcome this difficulty by complex analysis and the generation theorem of semigroups.

We notice that Mel'nikova [6] (also see [3, 7]) constructed a family of regularizing operators by using the solution of the well-posed Cauchy problem:

$$
v_{\varepsilon}^{\prime}(t)=\left(A-\varepsilon A^{2}\right) v_{\varepsilon}(t)(0<t \leq T), \quad v_{\varepsilon}(0)=x .
$$

Such a method is also called the quasi-reversibility method, introduced by Lattes and Lions 4]. Because (1.2) and (1.3) are different, the corresponding semigroup analysis and the construction of the family of regularizing operators in this paper are different from [6. One advantage is that (1.2) is related to the quasi-parabolic or Sobolev equation if $A$ is a realization of a partially differential operator (cf. 9]).

Throughout the paper, $B(X)$ is the space of all bounded linear operators from $X$ into itself. By $D(B), \sigma(B), \rho(B)$, and $R(\lambda, B)(\lambda \in \rho(B))$ we denote the domain, spectrum set, resolvent set, and resolvent of the operator $B$, respectively. Set $\Sigma_{\alpha}=\{\lambda \in \mathbf{C} \backslash\{0\} ;|\arg \lambda|<\alpha\}$ for $\alpha \in(0, \pi)$.

This paper is organized as follows. We prove in section 2 that $A(I+\varepsilon A)^{-1}-A$ generates a bounded analytic semigroup $\left\{E_{\varepsilon}(t)\right\}$ if $\{S(t)\}$ is bounded and $0 \in \rho(A)$. Moreover, there exists a positive constant $M$ independent of $\varepsilon$ such that $\left\|E_{\varepsilon}(t)\right\| \leq$ $M(t \geq 0)$. The main result of this paper is given in section 3 , which states that if $-A$ generates an analytic semigroup of angle $\alpha$ with $\pi / 4 \leq \alpha<\pi / 2$, then there exists a family of regularizing operators for the ill-posed Cauchy problem (1.1). In addition, Remark 3.3 points out that the restriction $\pi / 4 \leq \alpha<\pi / 2$ cannot be relaxed to $0<\alpha<\pi / 2$ by this paper's method.

\section{Semigroup analysis}

Let the operator $-A$ be a generator of a bounded analytic semigroup $S(t)$ of angle $\alpha, \pi / 4 \leq \alpha<\pi / 2$, and $0 \in \rho(A)$. For $\varepsilon>0$, set $J_{\varepsilon}=(I+\varepsilon A)^{-1}$ and $A_{\varepsilon}=A J_{\varepsilon}$. From the identity

$$
A_{\varepsilon}=\varepsilon^{-1}\left(I-J_{\varepsilon}\right)
$$

and from $J_{\varepsilon}$ being bounded, it follows that $A_{\varepsilon}$ is bounded. We thus can define a $C_{0}$-group of linear operators by

$$
S_{\varepsilon}(t)=\exp \left(-t A_{\varepsilon}\right), \quad-\infty<t<\infty,
$$

where we use the power series to define the exponential function.

By Theorem VII.9.5 in Dunford-Schwartz [1, p. 602],

$$
\sigma\left(A_{\varepsilon}\right)=\left\{\mu(1+\varepsilon \mu)^{-1} ; \mu \in \sigma(A)\right\} \cup\{1 / \varepsilon\} .
$$

So, $\Sigma_{2 \alpha} \subset \rho\left(-A_{\varepsilon}\right)$. For $\lambda \in \Sigma_{2 \alpha}$, we have

$$
\begin{aligned}
\left\|R\left(\lambda,-A_{\varepsilon}\right)\right\| & =\left\|(\lambda I+(1+\varepsilon \lambda) A)^{-1}+\frac{\varepsilon}{1+\varepsilon \lambda}\left\{I-\lambda(\lambda I+(1+\varepsilon \lambda) A)^{-1}\right\}\right\| \\
& =\left\|\varepsilon(1+\varepsilon \lambda)^{-1} I+(1+\varepsilon \lambda)^{-1}(\lambda I+(1+\varepsilon \lambda) A)^{-1}\right\| \\
& \leq \frac{\varepsilon}{|1+\varepsilon \lambda|}+\frac{M}{|\lambda \|| 1+\varepsilon \lambda \mid} \\
& \leq \frac{M}{|\lambda|}
\end{aligned}
$$


where the positive constant $M$ is independent of $\varepsilon$. Therefore, there exists a positive constant $M_{1}$ independent of $\varepsilon$ such that $\left\|S_{\varepsilon}(t)\right\| \leq M_{1}(t \geq 0)$. Also, it is not hard to show that $\overline{(I+A) D\left(A^{2}\right)}=X$. Noticing that $A_{\varepsilon}$ approximates $A$ on $D\left(A^{2}\right)$ for small $\varepsilon$, we have $\lim _{\varepsilon \rightarrow 0} S_{\varepsilon}(t) x=S(t) x$ for $x \in X$ by the approximation theorem of semigroups.

Theorem 2.1. For each $\varepsilon>0$ let $E_{\varepsilon}(t)=S(t) S_{\varepsilon}(-t)(t \geq 0)$. Then $E_{\varepsilon}(t)$ is an analytic semigroup on $X$ and $A_{\varepsilon}-A$ is its generator. In addition, there exists a positive constant $M$ independent of $\varepsilon$ such that $\left\|E_{\varepsilon}(t)\right\| \leq M(t \geq 0)$.

Proof. Similar to the proof of Lemma 1 in Showalter [9], $E_{\varepsilon}(t)$ is a semigroup on $X$ and $A_{\varepsilon}-A$ is the generator. Since $A_{\varepsilon}$ is bounded and $-A$ generates an analytic semigroup, $E_{\varepsilon}(t)$ is analytic by the perturbation theorem and the uniqueness of the semigroup.

In order to end the proof we first consider the spectrum of $A_{\varepsilon}-A$.

For $\lambda \in \mathbf{C}$, we have

$$
\begin{aligned}
\left(\lambda I-A_{\varepsilon}+A\right) x & =\left(\lambda I-\varepsilon^{-1} I+\varepsilon^{-1}(I+\varepsilon A)^{-1}+A\right) x \\
& =\left[\left(\lambda I-\varepsilon^{-1} I+A\right)(I+\varepsilon A)+\varepsilon^{-1}\right](I+\varepsilon A)^{-1} x \\
& =\left(\lambda I+\lambda \varepsilon A+\varepsilon A^{2}\right)(I+\varepsilon A)^{-1} x, \quad x \in D(A) .
\end{aligned}
$$

Denote $\Lambda=\sqrt{\varepsilon^{2} \lambda^{2}-4 \varepsilon \lambda}$, and take the branch of $\Lambda>0$ for $\varepsilon \lambda>4$. The inverse

$$
\begin{aligned}
& \left(\lambda I-A_{\varepsilon}+A\right)^{-1} \\
= & (I+\varepsilon A)\left(\lambda I+\lambda \varepsilon A+\varepsilon A^{2}\right)^{-1} \\
= & \varepsilon^{-1}(I+\varepsilon A)\left(A+\frac{\varepsilon \lambda-\Lambda}{2 \varepsilon} I\right)^{-1}\left(A+\frac{\varepsilon \lambda+\Lambda}{2 \varepsilon} I\right)^{-1} \\
= & \left(A+\frac{\varepsilon \lambda+\Lambda}{2 \varepsilon} I\right)^{-1}+\left(1-\frac{\varepsilon \lambda-\Lambda}{2}\right)\left(\varepsilon A+\frac{\varepsilon \lambda-\Lambda}{2} I\right)^{-1}\left(A+\frac{\varepsilon \lambda+\Lambda}{2 \varepsilon} I\right)^{-1} \\
= & \left(A-\frac{\varepsilon \lambda-\Lambda}{2 \varepsilon} I\right)^{-1}+\left(1-\frac{\varepsilon \lambda+\Lambda}{2}\right)\left(\varepsilon A+\frac{\varepsilon \lambda+\Lambda}{2} I\right)^{-1}\left(A+\frac{\varepsilon \lambda-\Lambda}{2 \varepsilon} I\right)^{-1}
\end{aligned}
$$

exists (as a bounded operator) if and only if

$$
-\frac{\varepsilon \lambda \pm \Lambda}{2 \varepsilon} \in \rho(A)
$$

This implies (see Figure 1)

$$
\sigma\left(A_{\varepsilon}-A\right) \subset\left\{-\varepsilon \lambda^{2}(1+\varepsilon \lambda)^{-1}: \lambda \in \sigma(A)\right\}:=\mathcal{G} .
$$

Set $\lambda=r e^{ \pm i(\pi / 2-\alpha)}$. Then the parametric equations of the curve $\gamma$ are as follows:

$$
\gamma:\left\{\begin{array}{l}
x=-\frac{\varepsilon r^{2}(\cos 2(\pi / 2-\alpha)+\varepsilon r \cos (\pi / 2-\alpha))}{1+2 r \varepsilon \cos (\pi / 2-\alpha)+\varepsilon^{2} r^{2}} \\
y=\mp \frac{\varepsilon r^{2}(\sin 2(\pi / 2-\alpha)+\varepsilon r \sin (\pi / 2-\alpha))}{1+2 r \varepsilon \cos (\pi / 2-\alpha)+\varepsilon^{2} r^{2}},
\end{array} \quad(r \geq 0),\right.
$$

where $\mathcal{G}$ is the left-side region surrounded by the curve $\gamma$.

For each $\beta \in[2(\pi / 2-\alpha), \pi / 2)$ or $\beta \in(\pi / 2,2 \alpha]$, it is not hard to show that the line $y=x \tan \beta$ intersects the curve $\gamma$ only at the origin. Hence,

$$
\Sigma_{2 \alpha} \subset \mathbf{C} \backslash \mathcal{G} \subset \rho\left(A_{\varepsilon}-A\right) .
$$





FigURE 1.

Now we estimate the resolvent. For $\lambda \in \Sigma_{2 \alpha} \subset \rho\left(A_{\varepsilon}-A\right)$, we have

$$
\left\|R\left(\lambda, A_{\varepsilon}-A\right)\right\| \leq \begin{cases}\left|\frac{2 \varepsilon}{\varepsilon \lambda+\Lambda}\right|+\left|1-\frac{\varepsilon \lambda-\Lambda}{2} \| \frac{2}{\varepsilon \lambda-\Lambda}\right|\left|\frac{2 \varepsilon}{\varepsilon \lambda+\Lambda}\right|, \quad \operatorname{Im} \lambda \geq 0, \\ \left|\frac{2 \varepsilon}{\varepsilon \lambda-\Lambda}\right|+\left|1-\frac{\varepsilon \lambda+\Lambda}{2}\right|\left|\frac{2}{\varepsilon \lambda+\Lambda}\right|\left|\frac{2 \varepsilon}{\varepsilon \lambda-\Lambda}\right|, \quad \operatorname{Im} \lambda<0 .\end{cases}
$$

Denote $\varepsilon \lambda$ by $z$, and define

$$
f(z)=\frac{z}{z+\sqrt{z^{2}-4 z}}
$$

for $z \in G_{+}:=\left\{z \in \Sigma_{2 \alpha} ; \operatorname{Im} z>0\right\}$ (see Figure 2). Then $f(z)$ is continuous in $G_{+}$.

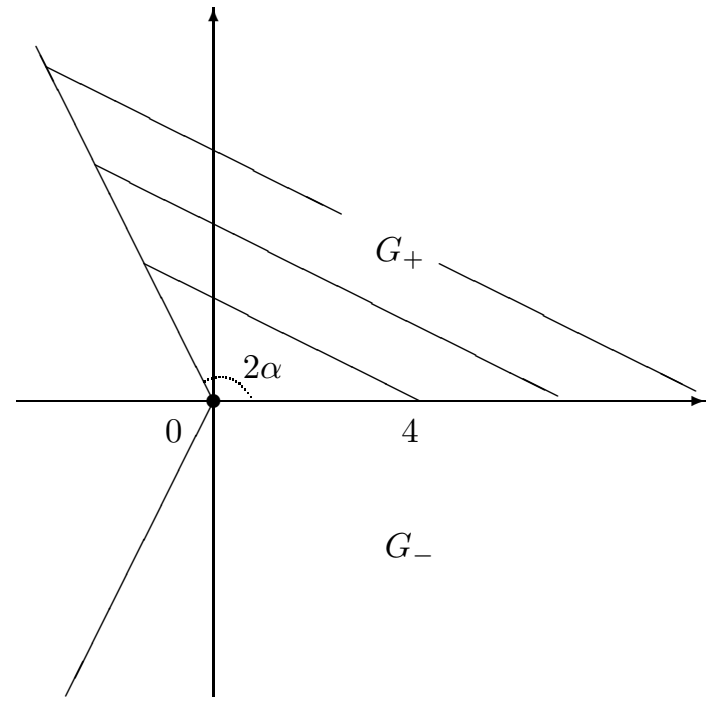

FiguRE 2. 
Moreover, $f(z)$ can be continuous to the upper segment of $[0,4]$. This means $f(z)$ is continuous on $\overline{G_{+}}$if we define $f(z)=0$ (since $f(z) \rightarrow 0$ as $|z| \rightarrow 0, \operatorname{Im} z>0$ ), where the upper segment of $[0,4]$ is a part of $\partial G_{+}$, the boundary of $G_{+}$.

Set $z=r e^{i \theta}, 0 \leq \theta \leq 2 \alpha$. Then

$$
\frac{\sqrt{z^{2}-4 z}}{z}=\frac{\sqrt{r^{2} e^{i 2 \theta}-4 r e^{i \theta}}}{r e^{i \theta}} \rightarrow 1(r \rightarrow \infty) .
$$

Consequently,

$$
\lim _{|z| \rightarrow \infty, z \in G_{+}} f(z)=\lim _{|z| \rightarrow \infty, z \in G_{+}}\left(1+\frac{\sqrt{z^{2}-4 z}}{z}\right)^{-1}=1 / 2 .
$$

Thus $f(z)$ is bounded on $\overline{G_{+}}$. Also, we have by l'Hospital's rule,

$$
\begin{aligned}
& \lim _{|z| \rightarrow \infty, z \in G_{+}} \frac{2}{z-\sqrt{z^{2}-4 z}}\left(1-\frac{z-\sqrt{z^{2}-4 z}}{2}\right) \\
= & \lim _{|z| \rightarrow \infty, z \in G_{+}} \frac{2-z+\sqrt{z^{2}-4 z}}{z-\sqrt{z^{2}-4 z}}=-1 .
\end{aligned}
$$

Moreover,

$$
\begin{aligned}
& \lim _{|z| \rightarrow 0, z \in G_{+}} \frac{2}{z-\sqrt{z^{2}-4 z}} \cdot \frac{2 z}{z+\sqrt{z^{2}-4 z}}\left(1-\frac{z-\sqrt{z^{2}-4 z}}{2}\right) \\
= & \lim _{|z| \rightarrow 0, z \in G_{+}}\left(1-\frac{z-\sqrt{z^{2}-4 z}}{2}\right) \\
= & 1 .
\end{aligned}
$$

Thus, the function

$$
g(z)=\left(1-\frac{z-\sqrt{z^{2}-4 z}}{2}\right) \cdot \frac{2 z}{z-\sqrt{z^{2}-4 z}} \cdot \frac{2}{z+\sqrt{z^{2}-4 z}}
$$

is bounded on $\overline{G_{+}}$, too. Consequently, there exists a positive constant $M$ such that

$$
|f(z)|+|g(z)| \leq M, \quad \operatorname{Im} z \geq 0 .
$$

Substituting $z$ for $\varepsilon \lambda$, it follows that

$$
\left\|R\left(\lambda, A_{\varepsilon}-A\right)\right\| \leq M /|\lambda|, \quad \operatorname{Im} \lambda \geq 0,
$$

for $\lambda \in \Sigma_{2 \alpha}$. Analogously, we have the same final estimate in $G_{-}$. In this case,

$$
\frac{\sqrt{\varepsilon^{2} \lambda^{2}-4 \varepsilon \lambda}}{\varepsilon \lambda}=\frac{\sqrt{\varepsilon^{2} r^{2} e^{i 2 \theta}-4 \varepsilon r e^{i \theta}}}{\varepsilon r e^{i \theta}} \rightarrow-1(r \rightarrow \infty)
$$

for $\lambda=r e^{i \theta},-2 \alpha<\theta<0$. This implies that $\sqrt{e^{i 2 \theta}}=\sqrt{e^{i(2 \theta+2 \pi)}}=-e^{i \theta}$.

Summing up,

$$
\left\|R\left(\lambda, A_{\varepsilon}-A\right)\right\| \leq M /|\lambda|, \quad \lambda \in \Sigma_{2 \alpha} .
$$

By the generation of semigroups, there exists a positive constant $M_{2}$ independent of $\varepsilon$ such that $\left\|E_{\varepsilon}(t)\right\| \leq M_{2}(t \geq 0)$. 


\section{Regularization for (1.1) AND A REMARK}

We start with the definition of a family of regularizing operators (cf. [10]).

Definition 3.1. A family $\left\{R_{\varepsilon, t} ; \varepsilon>0, t \in[0, T]\right\} \subset B(X)$ is called a family of regularizing operators for the problem (1.1) if for each solution $u(t)(0 \leq t \leq T)$ of (1.1) with initial element $x$, and for any $\delta>0$, there exists $\varepsilon(\delta)>0$, such that

(a) $\varepsilon(\delta) \rightarrow 0(\delta \rightarrow 0)$,

(b) $\left\|R_{\varepsilon(\delta), t} x_{\delta}-u(t)\right\| \rightarrow 0(\delta \rightarrow 0)$ for each $t \in[0, T]$ on the condition that $x_{\delta}$ satisfies $\left\|x_{\delta}-x\right\| \leq \delta$.

Define $R_{\varepsilon, t}=S_{\varepsilon}(-t)$ for $\varepsilon>0$ and $0 \leq t \leq T$, where $S_{\varepsilon}(t)$ is the semigroup generated by $-A_{\varepsilon}$. Then $\left\{R_{\varepsilon, t} ; \varepsilon>0, t \in[0, T]\right\} \subset B(X)$.

Theorem 3.2. Assume that $-A$ is the generator of an analytic semigroup of angle $\alpha, \pi / 4 \leq \alpha<\pi / 2$. Then the operator family $\left\{R_{\varepsilon, t}\right\}$ defined above is a family of regularizing operators for (1.1).

Proof. We first consider the case that $-A$ is the generator of a bounded analytic semigroup of angle $\alpha$ and $0 \in \rho(A)$, where $\pi / 4 \leq \alpha<\pi / 2$.

Let $u(t)(0 \leq t \leq T)$ be a solution of (1.1) with initial element $x$, and let $\left\|x_{\delta}-x\right\| \leq \delta$. By $\left\|A_{\varepsilon}\right\| \leq M \varepsilon^{-1}$, we have

$$
\left\|R_{\varepsilon, t}\right\|=\left\|S_{\varepsilon}(-t)\right\| \leq e^{M t / \varepsilon} \leq e^{M T / \varepsilon}, \quad t \in[0, T] .
$$

Choose $\varepsilon=-2 M T(\ln \delta)^{-1}$. Then $\varepsilon \rightarrow 0(\delta \rightarrow 0)$ and

$$
\left\|R_{\varepsilon, t} x_{\delta}-R_{\varepsilon, t} x\right\| \leq \delta e^{M T / \varepsilon}=\sqrt{\delta} \rightarrow 0(\delta \rightarrow 0) .
$$

The approximation theorem of semigroups with Theorem 2.1 and (2.1) yields that

$$
\lim _{\varepsilon \rightarrow 0} E_{\varepsilon}(t) x=x, \quad x \in X, \quad t \geq 0 .
$$

It is easy to show that $x=S(t) u(t)$. It follows that

$$
\left\|R_{\varepsilon, t} x-u(t)\right\|=\left\|S_{\varepsilon}(-t) S(t) u(t)-u(t)\right\|=\left\|E_{\varepsilon}(t) u(t)-u(t)\right\| \rightarrow 0(\delta \rightarrow 0) .
$$

Combining (3.1) with (3.2) yields that $\left\{R_{\varepsilon, t}\right\}$ is a family of regularizing operators for (1.1) by Definition 3.1.

Next, we return to the general case that $-A$ is the generator of an analytic semigroup. There exists a constant $\omega \in \mathbf{R}$ such that $\omega-A$ is the generator of a bounded analytic semigroup and $0 \in \rho(A-\omega)$. As seen above, there exists a family of regularizing operators $\left\{R_{\varepsilon, t}\right\}$ for the problem

$$
v^{\prime}(t)=(A-\omega) v(t)(0<t \leq T), \quad v(0)=x .
$$

Let $u(t)(0 \leq t \leq T)$ be a solution of (1.1) with initial element $x$. Then $v(t):=$ $e^{-\omega t} u(t)(0 \leq t \leq T)$ is a solution of (3.3) with initial element $x$. Hence for each $t \in[0, T]$,

$$
\left\|e^{\omega t} R_{\varepsilon(\delta), t} x_{\delta}-u(t)\right\|=e^{\omega t}\left\|R_{\varepsilon(\delta), t} x_{\delta}-v(t)\right\| \rightarrow 0(\delta \rightarrow 0),
$$

i.e. $\left\{e^{\omega t} R_{\varepsilon, t}\right\}$ is a family of regularizing operators for (1.1).

Remark 3.3. The method above is false for $0<\alpha<\pi / 4$. 



FIGURE 3.

Set $\varphi=\pi / 2-\alpha$. Consider the image of two rays $r \exp ( \pm i \varphi)(r \geq 0)$ under the map $\lambda \mapsto-\varepsilon \lambda^{2}(1+\varepsilon \lambda)^{-1}$. We consider only the case $\lambda=r \exp (i \varphi)$ by the symmetry.

It is easy to obtain that

$$
\frac{-\varepsilon(r \exp (i \varphi))^{2}}{1+\varepsilon r \exp (i \varphi)}=-\frac{\varepsilon r^{2}(\cos 2 \varphi+\varepsilon r \cos \varphi)}{1+2 \varepsilon r \cos \varphi+\varepsilon^{2} r^{2}}-i \frac{\varepsilon r^{2}(\sin 2 \varphi+\varepsilon r \sin \varphi)}{1+2 \varepsilon r \cos \varphi+\varepsilon^{2} r^{2}} .
$$

Denote the real part by $f(r)$, that is

$$
f(r)=-\frac{\varepsilon r^{2}(\cos 2 \varphi+\varepsilon r \cos \varphi)}{1+2 \varepsilon r \cos \varphi+\varepsilon^{2} r^{2}}, \quad r \geq 0 .
$$

It is obvious that $f(r) \leq 0$ for $0<\varphi \leq \pi / 4$.

When $\pi / 4<\varphi<\pi / 2, f^{\prime}(r)=0$ yields the following equation:

$$
r^{3} \varepsilon^{3} \cos \varphi+4 r^{2} \varepsilon^{2} \cos ^{2} \varphi+r \varepsilon(3+2 \cos 2 \varphi) \cos \varphi+2 \cos 2 \varphi=0 .
$$

Its unique real solution is

$$
r_{0}=\frac{1}{\varepsilon}\left(\frac{G^{1 / 3}}{3}-\frac{1-\frac{2}{3} \cos ^{2} \varphi}{G^{1 / 3}}-\frac{4}{3} \cos \varphi\right),
$$

where

$$
\begin{aligned}
& G=\left(8 \cos ^{4} \varphi-36 \cos ^{2} \varphi+27+3 H\right) / \cos \varphi, \\
& H=\sqrt{-48 \cos ^{6} \varphi+180 \cos ^{4} \varphi-213 \cos ^{2} \varphi+81} .
\end{aligned}
$$

This suggests that $f\left(r_{0}\right)=C / \varepsilon=\max _{r \geq 0} f(r)$, where $C=\left.f\left(r_{0}\right)\right|_{\varepsilon=1}>0$ for $\pi / 4<\varphi<\pi / 2$. We thus understand the range of the spectrum $\sigma\left(A_{\varepsilon}-A\right)$ (see the shadow part of the right side in Figure [3). In view of the above-mentioned facts, it is not necessary to apply the approximation theorem of semigroups to $\lim _{\varepsilon \rightarrow 0} E_{\varepsilon}(t)$. In fact, although $A_{\varepsilon}-A$ generates an analytic semigroup, its growth order $\omega$ is dependent on $\varepsilon$ (indeed, $\omega=M / \varepsilon$ for some positive constant $M$ ). This indicates 
that it may be impossible to relax the restriction $\pi / 4 \leq \alpha<\pi / 2$ to $0<\alpha<\pi / 2$ by this paper's method.

\section{REFERENCES}

[1] N. Dunford and J. T. Schwartz, Linear Operators, Part I, Interscience, New York, 1985. MR 1009162 (90g:47001a)

[2] H. Gajewski and K. Zacharias, Zur Regularisierung einer Klasse nichtkorrekter Probleme bel Evolutionsgleichungen, J. Math. Anal. Appl. 38(1972), 784-789. MR0308625 (46:7739)

[3] V. K. Ivanov, I. V. Mel'nikova and A.I. Filinkov, Differential Operator Equations and Illposed Problems, Nauka, Moscow, 1995. (In Russian) MR 1415388 (97j:47092)

[4] R. Lattes and J.-L. Lions, The Method of Quasi-reversibility Applications to Partial Differential Equations, Amer. Elsevier Publ. Co., New York, 1969. MR0243746 (39:5067)

[5] R. deLaubenfels, Existence Families, Functional Calculi and Evolution Equations, SpringerVerlag, Berlin, 1994. Mr.1290783 (96b:47047)

[6] I. V. Mel'nikova, General theory of the ill-posed Cauchy problem, J. Inverse and Ill-posed Problems 3(1995), 149-171. MR1341036 (97a:34156)

[7] I. V. Mel'nikova and A.I. Filinkov, Abstract Cauchy Problems: Three Approaches, Chapman and Hall, London, 2001. MR 1823612 (2002h:34110)

[8] A. Pazy, Semigroups of Linear Operators and Applications to Partial Differential Equations, Springer-Verlag, Berlin, 1983. MR.0710486 (85g:47061)

[9] R. E. Showalter, The final problem for evolution equations, J. Math. Anal. Appl. 47(1974), 563-572. MF 0352644 (50:5131)

[10] A. N. Tikhonov and V. Y. Arsenin, Solution of Ill-posed Problems, Winston and Sons, Washington D.C., 1977. MR0455365|(56:13604)

Department of Mathematics, Huazhong University of Science and Technology, Wuhan 430074, People's Republic of China

E-mail address: huang5464@hotmail.com

Department of Mathematics and Center for Optimal Control and Discrete Mathematics, Huazhong Normal University, Wuhan 430079, People's Republic of China - And Department of Mathematics, Huazhong University of Science and Technology, Wuhan 430074, People's Republic of China

E-mail address: qzheng@hust.edu.cn 